Bio - grafia. Escritos sobre la Biología y su Enseñanza. ISSN 2027

Edición Extraordinaria. p.p. 1601-1613

Memorias del VIII Encuentro Nacional de Experiencias en Enseñanza de la Biología y la Educación Ambiental. III Congreso Nacional de Investigación en Enseñanza de la Biología.

\title{
CAMBIO Y LA PERMANENCIA EN LOS FENÓMENOS ASOCIADOS A LA METAMORFOSIS, GUIAS DIDÁCTICAS PARA NIÑOS DE GRADO CUARTO: REFLEXIONES SOBRE LA PRODUCCIÓN DE CONOCIMIENTO DE LOS MAESTROS DE CIENCIAS
}

\begin{abstract}
About the change and stay in the phenomena associated with metamorphosis guidelines for teaching children of fourth grade: reflections on the production of knowledge of science teachers
\end{abstract}

Rodríguez Yudy: Sánchez David²; Silva Álvaro³.

RESUMEN: Esta investigación es producto de una serie de cuestionamientos que surgieron de la reflexión que sobre su propio conocimiento escolar tienen los docentes de ciencias a propósito de la producción de guías didácticas que permitan construir explicaciones sobre el cambio y la permanencia de los fenómenos asociados a la metamorfosis de los lepidópteros. Se evidencia como las tensiones y complejizaciones de los docentes sobre su práctica educativa y su conocimiento escolar, generan complejas, sistemáticas y diferentes formas de trabajo, recorriendo categorías que van de lo mágico religioso, pasando por explicaciones mecanicista, hasta construcciones relacional-emergentes. Se pretenden direccionar una ruta para la producción de sentido escolar en estudiantes, cuestionar lo que cambia y lo que permanece con guias tituladas: a- Animado y animado única mirada, b- la mecánica de la metamorfosis, c- Emergencia, el simbolismo de lo complejo. También se muestra un panorama sobre la forma en que maestros y estudiantes recorren campos de producción de conocimiento análogo al surgimiento de las ciencias naturales, complejizado sus propias explicaciones, desde una mirada ingenua determinada y visible propia de las relaciones sensibles con el entorno hacia la explicación naturalista y vinculante de sustancias, con sus interacciones, relaciones, transformaciones y emergencias que permiten

\footnotetext{
${ }^{1}$ Estudiante maestría en docencia de las ciencias naturales, Universidad Pedagógica Nacional. yudyrodriguez1989@gmail.com

2 Docente investigador Postgrados Universidad pedagogica Nacional. dsanchez.david@gmail.com

${ }^{3}$ Estudiante maestría en docencia de las ciencias naturales, Universidad Pedagógica Nacional. aulasvivas_11@hotmail.com
} 
Bio - grafia. Escritos sobre la Biología y su Enseñanza. ISSN 2027

Edición Extraordinaria. p.p. 1601-1613

Memorias del VIII Encuentro Nacional de Experiencias en Enseñanza de la Biología y la Educación Ambiental. III Congreso Nacional de Investigación en Enseñanza de la Biología.

explicar la metamorfosis: a- una experiencia sensible, b- detallando lo visible. Una postura naturalista, c- mirada constructiva y reconstructiva, la reflexión y resignificación.

PALABRAS CLAVES: Cambio y permanencia, metamorfosis, lepidópteros, construcción de explicaciones.

ABSTRACT: This investigation stems from a series of questions that emerged from the reflection on their own knowledge school science teachers have about the production of tutorials that build explanations of change and permanence of the phenomena associated with the metamorphosis Lepidoptera. It is evident as tensions and complejizaciones of teachers on their teaching practice and school knowledge, it generates complex, systematic and different forms of work, covering categories ranging from the magical religious, through mechanistic explanations, to relational-emerging buildings are intended to address a route for the production of sense school students intend to question what changes and what stays with guides entitled: Lively and animated a- unique look, bmechanics metamorphosis, c- Emergency symbolism complex. An overview of how teachers and students roam production fields similar knowledge to the emergence of the natural sciences is also displayed, more complex their own explanations, from a certain naive look and own visible sensitive relations with the environment toward explaining naturalist and binding substances, their interactions, relationships, transformations and emergencies to explain the metamorphosis: a- a sensitive experience, b- detailing the visible. A naturalistic stance, $c$ - constructive and reconstructive eye, reflection and redefinition

Keys word: Change and permanence, metamorphosis lepidoptera, construction of explanations.

\section{INTRODUCCIÓN:}

¿Qué ocurre al interior de un organismo o al interior de un huevo cuando se está gestando a un ser? ¿Es un designio divino el que se expresa en tal perfección?, ¿Qué organizaciones en las partes que constituyen los organismos originan tales formas? ¿Estará preformado ese ser o sus partes se desarrollan paulatinamente hasta su emergencia al mundo exterior?, reflexiones como estas el inicio de encuentros y desencuentros desde donde los maestros 
Bio - grafia. Escritos sobre la Biología y su Enseñanza. ISSN 2027

Edición Extraordinaria. p.p. 1601-1613

Memorias del VIII Encuentro Nacional de Experiencias en Enseñanza de la Biología y la Educación Ambiental. III Congreso Nacional de Investigación en Enseñanza de la Biología.

de la maestría en docencia de las ciencias edifican y construyen sus intervenciones de aula alrededor del cambio y la permanencia de los organismos.

Por lo general, cuando enseñamos ciencias; marcados por una visión unicausal, donde el "cambio o lo que permanece en un organismo" se determina cuando se evidencia la modificación de tamaño y formas de las estructuras externas que por efecto varía su funcionalidad; de tal forma que el fenómeno entra al terreno de la obviedad y las reflexiones por las relaciones, sustancias, transformaciones presentes en el proceso no tienen relevancia pues requieren ser demostradas; se reproduce, no se produce, se memoriza, no se comprende, se determina, no se construye. Será necesario entonces cambiar esta idea sobre la enseñanza, pensar ahora que es lo indispensable para la construcción de conocimiento sobre lo vivo en la escuela. Integrar al estudiante y al docente en un escenario en el cual se asuma al conocimiento científico como uno de los elementos, no el único y necesario para explicar lo que nos rodea, será necesario ahora promover espacios desde los cuales sus propios saberes sean significantes, se significan en la experiencia con los otros y con el objeto, se reconstruyen en las metáforas, analogías símiles y explicaciones que emergen de la producción de sentido. Consideramos estos elementos esenciales para que la actividad de construcción de conocimiento en ciencias adquiera sentido.

\section{METODOLOGÍA}

El trabajo toma como base la investigación cualitativa, entendida desde Taylor y Bogdan (1996) como aquella que se realiza al interior de la situación estudiada, captando una imagen de lo que dicen y hacen las personas, se deja que las palabras y las acciones hablen por sí mismas, realizando una comprensión e interpretación de fenómenos Con la intención de proporcionar herramientas que conformen campos de análisis sobre las formas en que los sujetos constituyen una actividad de construcción de conocimiento alrededor de un fenómeno particular; la permanencia y cambio de los organismos desde el trabajo con estudiantes de grado 4 (con edades que oscilan entre los 8 y 10 años) de un colegio perteneciente a la zona urbana de la ciudad de Bogotá y docentes en formación del programa de maestría en Docencia de las Ciencias. 
Edición Extraordinaria. p.p. 1601-1613

Memorias del VIII Encuentro Nacional de Experiencias en Enseñanza de la Biología y la Educación Ambiental. III Congreso Nacional de Investigación en Enseñanza de la Biología.

Este proceso contempla: 1. Construcción de la estrategia de aula. En su desarrollo y elaboración se han conformado 3 momentos distintos desde los cuales se ha explicado el fenómeno de la permanencia y el cambio en los organismos, partiendo de algunas construcciones explicativas realizadas desde las ciencias para dar cuenta de las transformaciones sufridas por los insectos. 2. Descripción de la ruta de observación de los resultados.

\section{CONSTRUCIÓN DE LA ESTRATEGIA DE AULA}

Durante todo el trabajo se reconocen tres grandes instancias que permiten la comprensión de la evolución y complejización de las explicaciones de los estudiantes sobre el fenómeno de cambio y permanencia en las transformaciones sufridas por los insectos en el proceso de metamorfosis. Este tránsito se transformó en una dialéctica constante entre la revisión y constitución del campo de conocimiento para la disciplina a nivel histórico-epistemológico como una gran traducción pedagógica derivada de los docentes en dos grandes aspectos: metodológicos y teóricos; de tal manera que dichas reflexiones posibiliten no solo la construcción de la estrategia de aula, sino también la emergencia de las rutas de observación y análisis para dar cuenta de las explicaciones generadas por docentes y estudiantes implicados en esta experiencia. Este recorrido conceptual se configura desde Valencia, et al, 2000; Wilson, 2007; Mayr, 1995; Jacob, 1984; Jacob, 1998 y a su vez relacionados con la reflexión del maestro en su quehacer en el aula. (Ver gráfico 1).

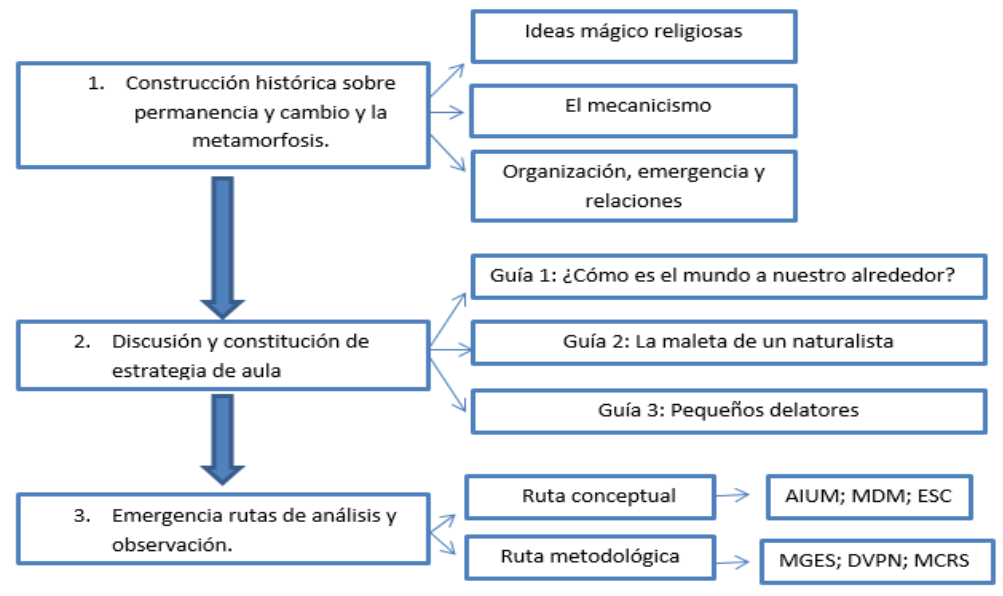


Bio - grafia. Escritos sobre la Biología y su Enseñanza. ISSN 2027

Edición Extraordinaria. p.p. 1601-1613

Memorias del VIII Encuentro Nacional de Experiencias en Enseñanza de la Biología y la Educación Ambiental. III Congreso Nacional de Investigación en Enseñanza de la Biología.

\section{Grafico $N^{\circ} 1$ Fases de trabajo}

Un primer momento implico la revisión histórica y epistemológica, en ella se reconoce inicialmente lo que denominamos "la mirada mágico religiosa", en esta instancia se encuentran explicaciones en donde se podrían reconocer elementos Animistas que según la literatura revisada no muestran una clara distinción entre lo vivo y lo inanimado, en esta mirada se centro la primera unidad de observación, desde donde se organizaron las primeras reflexiones alrededor de las explicaciones posibles derivadas de actividades intencionadas sobre preguntas de eventos naturales cotidianos como el día y la noche, los cambios visibles en la luna y otros. Todos estor artilugios no tenían otra intención que reconocer las formas que usan los estudiantes para dar cuenta sus primeras y generales miradas a propósito de lo que podría cambiar o permanecer en cada situación relacionada con la metamorfosis. Esta estrategia se organizó en forma de guía (1) ¿Cómo es el mundo a nuestro alrededor? En ella se describen situaciones que muy seguramente vivieron los primeros hombres frente a los fenómenos de la naturaleza, se plantean sus posibles explicaciones y se cuestiona a los estudiantes sobre sus propias explicaciones a propósito de lo que cambia y permanece en fenómenos macro de la naturaleza, el día y la noche, las estaciones, los cambios en el relieve y el paisaje, hasta los fenómenos macros de las transformaciones de los sistemas vivo como la metamorfosis de los insectos y los anfibios, todo este trabajo busca de alguna manera evidenciar los mitos, miradas ingenuas, explicaciones generales y demás aspectos que den cuanta de ideas animistas sobre los fenómenos de cambio y permanencia de los fenómenos naturales incluida la metamorfosis, aquí la ruta explicativa es mágica, mítica e incluso espiritual, refleja un poco el nacimiento de las primeras descripciones sobre fenómenos naturales, recapitula la soberanía de la explicación vitalista de los fenómenos naturales

En un segundo momento emerge la idea de como explicar los fenómenos naturales mas desde la pregunta, desde la observación detallada y meticulosa de la naturaleza que del mito; seguramente emulando a los grandes naturalistas como Humboldt, esta nueva aproximación a los fenómenos de cambio y permanecía, permiten entre otros aspectos, detallar la observación de las estructuras y establecer nexos entre ellas como si se trataran de grandes y eficientes maquinas, esta aproximación al modelo mecanicista desde una mirada histórico natural, privilegia la descripción detallada de las partes visibles y su 
Bio - grafia. Escritos sobre la Biología y su Enseñanza. ISSN 2027

Edición Extraordinaria. p.p. 1601-1613

Memorias del VIII Encuentro Nacional de Experiencias en Enseñanza de la Biología y la Educación Ambiental. III Congreso Nacional de Investigación en Enseñanza de la Biología.

funcionamiento incluso con leyes de la fisicoquímicas sobre los procesos de cambio y permanencia en la metamorfosis, estas ideas se van plasmando en la en la guía $\mathrm{N}^{\circ} 2$ de trabajo titulada la maleta de un naturalista. En donde se incita al estudiante a actuar y pensar como un naturalista, observando juiciosa y detalladamente el fenómeno de la metamorfosis y asi determinar las probales explicaciones de cambio y permanencia que allí acurren, en esta guía se busca dibujar y explicar los detalles tal y como lo haría un naturalista, las diferencias entre huevos, larvas pupas y adultos en sus sucesivas transformaciones, todo esto obliga al estudiante a centrarse en el ciclo de vida, de un insecto o de un anfibio, se exploran los diferentes tipos de metamorfosis, completas, incompletas y se establecen las principales semejanzas y diferencias como si se buscaran leyes universales que pudieran explicar los fenómenos de cambio y permanencia asociados al fenómenos de metamorfosis de cualquier animal (anfibio e insecto) este sincretismo entre la explicación mecanicista y la descripción naturalista permite un nuevo orden de explicaciones en el aula de ciencias, que ya no depende de la intuición o de la magia.

En un momento final, la ruta de trabajo trasciende las explicaciones mecanicista y su acercamiento desde modelos naturalistas del mundo, ahora se identifican aspectos relacionados con la organización, la emergencia de modelos simbólicos y como cualquier postura cientifista contemporánea, se incluye el método como protocolo para la comprensión y explicación de los fenómenos naturales, además se complejiza el discurso de la metamorfosis al incluir variables externas y sinérgicas que se permiten la pregunta por lo interno, lo no visible, lo no necesariamente causal, desde otra explicación diferente a la magia. Se espera entonces una explicación del cambio y la permanencia de los fenómenos de metamorfosis desde las transformaciones mismas, el cambio en las sustancias y relaciones de las hormonas o el sistema endocrino, el cambio en los procesos de desarrollo y crecimiento en el ciclo de vida de los insectos, explicaciones mas sistémicas están ahora emergiendo, estas nuevas ideas son traducidas en la guía $n^{\circ} 3$ titulada "Pequeños Delatores". Esta ultima ruta de trabajo aprovecha la entomología forense para formular por ejemplo, la pregunta por las diferencias en tiempos y movimientos de cada ciclo de vida asociados a la metamorfosis de diversos insectos, adentra en las explicaciones sobre las hormonas y sus papel en los cambios de estadios en la metamorfosis, cuestiona el papel de los factores externos como el clima, la temperatura, o las condiciones de luz y sombras en 
Bio - grafia. Escritos sobre la Biología y su Enseñanza. ISSN 2027

Edición Extraordinaria. p.p. 1601-1613

Memorias del VIII Encuentro Nacional de Experiencias en Enseñanza de la Biología y la Educación Ambiental. III Congreso Nacional de Investigación en Enseñanza de la Biología.

las transformaciones que ocurren durante el fenómeno de la metamorfosis. El recorrido es ahora por las explicaciones menos obvias, mas elaboradas desde la fisicoquímica, del metabolismo, será necesario seguirle la pista a los insectos y sus cambios desde la ciencia moderna, la entomología forense, el análisis de la metamorfosis en un laboratorio, ya no describiendo partes y relaciones, ahora macerando y extrayendo sustancias químicas que en determinadas concentraciones pueden dar cuenta del cambio y la permanencia para finalmente llegar al mariposario como posibilidad de comprender fenómenos naturales en ambientes articifializados como son las ciudades, todo en un modelo de simulación de condiciones para producir nuevas re significaciones de sentido

\begin{tabular}{|c|l|}
\hline GUÍA & Momentos conceptuales e históricos \\
\hline $\begin{array}{c}\text { Guía 1. ¿Cómo es el } \\
\text { mundo a nuestro } \\
\text { alrededor? }\end{array}$ & $\begin{array}{l}\text { La experiencia sensible (de primera mano con el fenómeno de cambio y } \\
\text { permanencia) desde miradas mágico religiosas. }\end{array}$ \\
\hline $\begin{array}{c}\text { Guía 2. La maleta de un } \\
\text { naturalista. }\end{array}$ & $\begin{array}{l}\text { El privilegio por formas, estructuras visibles, colores, partes, desde los } \\
\text { postulados mecanicistas, apoyado en la observación detallada lo visible. }\end{array}$ \\
\hline $\begin{array}{c}\text { Guía 3. Pequeños } \\
\text { delatores }\end{array}$ & $\begin{array}{l}\text { Comprensión de la dinámica interna del organismo, desde sustancias, } \\
\text { transformaciones, nuevas emergencias y el proceso de artificialización, } \\
\text { desde una mirada reconstructiva. }\end{array}$ \\
\hline
\end{tabular}

Tabla $N^{\circ} 1$ Resumen de los momentos conceptuales y metodológicos asociados a las tres guías de trabajo.

\section{DESCRIPCIÓN DE LA RUTA DE OBSERVACION DE LOS RESULTADOS}

Las rutas de observación y análisis de resultados se construyeron teniendo en cuenta el recorrido histórico y conceptual, desde los cuales se pretende observar la manera como configuraron explicaciones los estudiantes sobre la permanencia y cambio en los sistemas vivientes. 
Bio - grafia. Escritos sobre la Biología y su Enseñanza. ISSN 2027

Edición Extraordinaria. p.p. 1601-1613

Memorias del VIII Encuentro Nacional de Experiencias en Enseñanza de la Biología y la Educación Ambiental. III Congreso Nacional de Investigación en Enseñanza de la Biología.

El eje de análisis metodológico abarca las formas de aproximación al cambio y la permanencia en la metamorfosis desde un aumento en la complejidad de observación del fenómeno, de esta manera estas rutas de observación se denominaron: (MGES) Mirada gruesa la experiencia sensible; (DVMN) Detallar los visible la mirada naturalista y finalmente (MCRS) Mirada constructiva y reconstructiva "la reflexión y re significación. Estas categorías que se observan en todo el momento de la investigación son producto de diferentes análisis; por un lado la re significación histórico y epistemológica del fenómenos de metamorfosis a lo largo de la historia de las ciencias, por otro lado, las tensiones y discusiones de los docentes sobre su propio saber disciplinar en la búsqueda de un nuevo saber escolar para la enseñanza del el cambio y permanencia asociado al fenómeno de la metamorfosis y finalmente del análisis de la complejizacion de explicaciones en los estudiantes durante el desarrollo de la propuesta de aula

De una forma general, la categoria MGES pretende identificar las ideas contemplativas y someras que sobre el fenómeno de la metamorfosis, cambio y permanencia tienen los estudiantes; las explicaciones desde la experiencia que tenemos de primera mano con el evento, es decir, desde el asombro y la curiosidad en su forma más sencilla.

La categoría DVMN, pretende dilucidar las ideas desde la cuales los estudiantes logran realizar descripciones detalladas del fenómeno mediante observaciones detalladas y más concisas de las estructuras asociadas a la metamorfosis; permite pensar el cambio y permanencia en la desde las leyes físicas, donde se hace necesario definir y describir las formas visibles y establecer sus funciones, es decir el privilegio de las estructuras por encima de las interacciones.

Y en la categoría MCRS, se intentan cuestionar la experiencia cotidiana y las formas más simples de la observación, posibilitando la formulación de nuevas preguntas y formas de aproximación al fenómeno natural estudiado, de esta manera construir diferentes explicaciones sobre cambio y permanencia a propósito de la metamorfosis. Se asume el cambio y permanencia en la metamorfosis como una relación entre el entorno y el organismo, como un proceso que constituye las dinámicas, funcionamientos, emergencias y transformaciones de los constituyentes de lo vivo. 
Bio - grafia. Escritos sobre la Biología y su Enseñanza. ISSN 2027

Edición Extraordinaria. p.p. 1601-1613

Memorias del VIII Encuentro Nacional de Experiencias en Enseñanza de la Biología y la Educación Ambiental. III Congreso Nacional de Investigación en Enseñanza de la Biología.

Estas rutas de observación permitieron realizar el análisis de los diferentes hallazgos luego de implementar la estrategia de aula, con los datos obtenidos mediante grabaciones de audio y video, análisis de contenido de lo escrito y expresado por los estuantes en las guias y reconfiguración de sentido por parte de los docentes investigadores.

\section{RESULTADOS Y DISCUSIÓN PARCIAL}

Se observa como estudiantes y maestros han organizado múltiples interpretaciones sobre el cambio y permanencia en la metamorfosis de los insectos. Unas vinculan fuerzas ocultas que actúan y dirigen el devenir de las cosas, con un sentido y un fin propuesto por una fuerza desconocida, explicando el fenómeno desde la experiencia sensible que puede proporcionar los sentidos, se asume que las transformaciones son estados progresivos a un estado superior, es decir, el insecto imperfecto (larva) y el perfecto (mariposa), entre otras. Desde esta mirada se reconoce lo que cambia y permanece en la metamorfosis, surge la evidencia de lo visible, las explicaciones eventualmente acuden a reconocer la estructura y composición de las partes como recurso necesario para comprender el evento.

Otro tipo de interpretaciones centran sus esfuerzos en reconocer detalladamente la composición y estructura de los organismos como el principal recurso interpretativo y explicativo que permite dar cuenta de lo que cambia y lo que permanece en el proceso de la metamorfosis de los insectos. Por último se encuentran las que consideran y vinculan la descripción de estructuras, sustancias, condiciones presentes y las transformaciones, como elementos que se relacionan para configurar emergencias apreciables en las modificaciones drásticas presentes en la metamorfosis. Se aclara que las explicaciones de los integrantes que se pueden ubicar en este último momento no se encuentran presentes en la totalidad del grupo, existen casos particulares que se pueden ubicar allí. De modo que desde esta reflexión se puede reconocer lo complejo que se presenta la comprensión de este fenómeno, tanto para el conocimiento construido desde las ciencias, como también para estudiantes y docentes que hacen parte de esta experiencia.

Las categorías que surgieron de un primer análisis fueron:

\begin{tabular}{|c|c|c|c|}
\hline $\begin{array}{c}\text { "mirada gruesa, } \\
\text { la EXPERIENCIA } \\
\text { SENSIBLE } \\
\text { (MGES) }\end{array}$ & $\begin{array}{c}\text { a. La metamorfosis como } \\
\text { expresión de fuerzas } \\
\text { ocultas. } \\
\text { b. Antropomorfismo en } \\
\text { alquianestada de la } \\
\text { metamorfosis asociada a } \\
\text { un designio divino. } \\
\text { c. Los estados de la } \\
\text { metamorfosis como } \\
\text { pupas y mariposas } \\
\text { asociadas a la vida y } \\
\text { muerte deulos humanos. }\end{array}$ & $\begin{array}{c}\text { a. La metamorfosis como un } \\
\text { proceso armonioso } \\
\text { sinónimo de belleza y } \\
\text { perfección } \\
\text { b. La metamorfosis } \\
\text { observada como un } \\
\text { proceso no lineal. } \\
\text { c. Descripción gradual y } \\
\text { detallada aún desde lo } \\
\text { externo de huevos, } \\
\text { larvas, pupas y adultos. }\end{array}$ & $\begin{array}{l}\text { a. Transformaciones en } \\
\text { cada uno de los } \\
\text { estadios de la } \\
\text { metamorfosis como } \\
\text { eventos independientes } \\
\text { de las condiciones } \\
\text { ambientales. } \\
\text { b. Adaptaciones de los } \\
\text { huevos, larvas, pupas y } \\
\text { mariposas a las } \\
\text { condiciones sin } \\
\text { visualización de lo } \\
\text { interno. }\end{array}$ \\
\hline
\end{tabular}


Memorias del VIII Encuentro Nacional de Experiencias en Enseñanza de la Biología y la Educación Ambiental. III Congreso Nacional de Investigación en Enseñanza de la Biología.

En la primera categoría se evidencia que vinculan fuerzas ocultas que actúan y dirigen el devenir de las cosas, con un sentido y un fin propuesto por una entidad superior, el observador se limita a contemplar el fenómeno porque en él está representado la perfección de su creador, las transformaciones drásticas sufridas por algunos organismo se análoga como una transición hacia un estado superior, el insecto imperfecto (larva) y el perfecto (mariposa). La expresión de estas fuerzas sobrenaturales se encuentra en un todo, desde el origen de los seres y las cosas, su acontecer y su finalidad.

\begin{tabular}{|c|c|c|c|}
\hline $\begin{array}{c}\text { Detallar los } \\
\text { visible, la } \\
\text { Postura } \\
\text { naturalista } \\
\text { (DVPN) }\end{array}$ & $\begin{array}{c}\text { a. El cambio y la } \\
\text { permanencia de la } \\
\text { metamorfosis como una } \\
\text { creación mantenida o } \\
\text { prolongada por un bien } \\
\text { divino. } \\
\text { b. La metamorfosis como } \\
\text { una alusión a la escala } \\
\text { natural (perfección). } \\
\text { c. Huevos y pupas como } \\
\text { estadios inanimados, } \\
\text { larvas y mariposas como } \\
\text { estadios animados. }\end{array}$ & $\begin{array}{c}\text { a. Descripción detallada de } \\
\text { huevos, pupas, larvas y } \\
\text { adultos desde la } \\
\text { estructura visible y sus } \\
\text { relaciones entre } \\
\text { componentes. } \\
\text { b. La comparación entre } \\
\text { estructuras de los } \\
\text { estadios presentes en el } \\
\text { ciclo de vida como } \\
\text { elementos para } \\
\text { comprender mecanismos } \\
\text { de desarrolla y } \\
\text { transformación. } \\
\text { Descripción inicial de los } \\
\text { mecanismos del ciclo de } \\
\text { vida en disposición de las } \\
\text { partes y sustancias como } \\
\text { forma de organizar la } \\
\text { estructura. (inicio de la } \\
\text { mecánica interna). }\end{array}$ & $\begin{array}{c}\text { a. Sustancia y eventos } \\
\text { internos en la } \\
\text { explicación de los } \\
\text { cambios de la } \\
\text { metamorfosis. } \\
\text { Sustancias y } \\
\text { mecanismos internos } \\
\text { como modelo de } \\
\text { explicación del control } \\
\text { de emergencias de } \\
\text { relaciones. } \\
\text { b. Explicación de los } \\
\text { mecanismos que } \\
\text { inhiben o alteran la } \\
\text { metamorfosis a partir } \\
\text { sustancias. } \\
\text { c. Explicación de los } \\
\text { mecanismos de } \\
\text { inhibición o alteración } \\
\text { de hormonas y } \\
\text { sustancias. }\end{array}$ \\
\hline
\end{tabular}

De esta manera, la preocupación por las descripciones permite establecer algunas ideas sobre los mecanismos del ciclo de vida en disposición de las partes y sustancias como

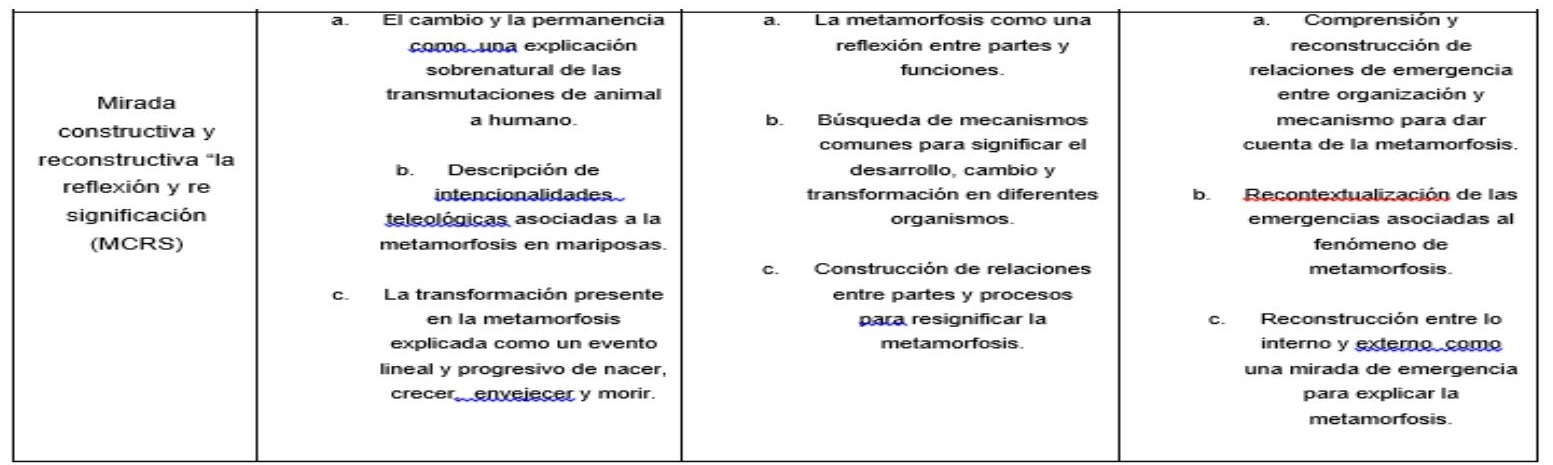


Bio - grafia. Escritos sobre la Biología y su Enseñanza. ISSN 2027

Edición Extraordinaria. p.p. 1601-1613

Memorias del VIII Encuentro Nacional de Experiencias en Enseñanza de la Biología y la Educación Ambiental. III Congreso Nacional de Investigación en Enseñanza de la Biología.

forma de organizar la estructura, Se establece una relación entre lo que come el organismo y su estructura, además se asume la presencia posible de sustancias que modifican estas estructuras. Así mismo, se atribuye una forma de reserva del organismo que se supone gasta en todo el proceso de metamorfosis.

En esta última instancia se vinculan elementos que proporcionan nuevos campos problematizadores en donde la preocupación por la descripción de las partes como recurso explicativo deja de ser suficiente, para trasladarse a la pregunta por las relaciones y las condiciones que hacen posible el proceso de la metamorfosis.

\section{Reflexiones finales}

Uno de los problemas que circulan en torno al cambio y la permanencia de los organismos está vinculado íntimamente a que es asumida como un fenómeno de estricta causalidad. Numerosas investigaciones se han desprendido alrededor de este campo, dejan ver a la este evento como un fenómeno complejo en donde intervienen múltiples factores.

Para este grupo de investigación, reconoce en esta experiencia una constante reflexión con los saberes propios, los construidos por la ciencia y la relación establecida y replanteada para reconocer en el evento una complejidad para su comprensión, a este ejercicio se denominó "el juego en Múltiples terrenos para explicar la metamorfosis": 
Bio - grafia. Escritos sobre la Biología y su Enseñanza. ISSN 2027

Edición Extraordinaria. p.p. 1601-1613

Memorias del VIII Encuentro Nacional de Experiencias en Enseñanza de la Biología y la Educación Ambiental. III Congreso Nacional de Investigación en Enseñanza de la Biología.

El primero tiene que ver con la observación e indagación desde una experiencia de base. En nuestro caso fue la experiencia de primera mano o experiencia sensible

El segundo está ligado al recurrir a la construcción de explicaciones realizadas desde la ciencia, respecto a la metamorfosis de los insectos.

Y el tercero tiene que ver con la Interpretación y construcción de explicaciones desde los dos terrenos mencionados anteriormente, poniéndolos a prueba en la observación y análisis del evento en condiciones particulares intentando de este modo una complejización de nuestra manera misma de comprender lo vivo.

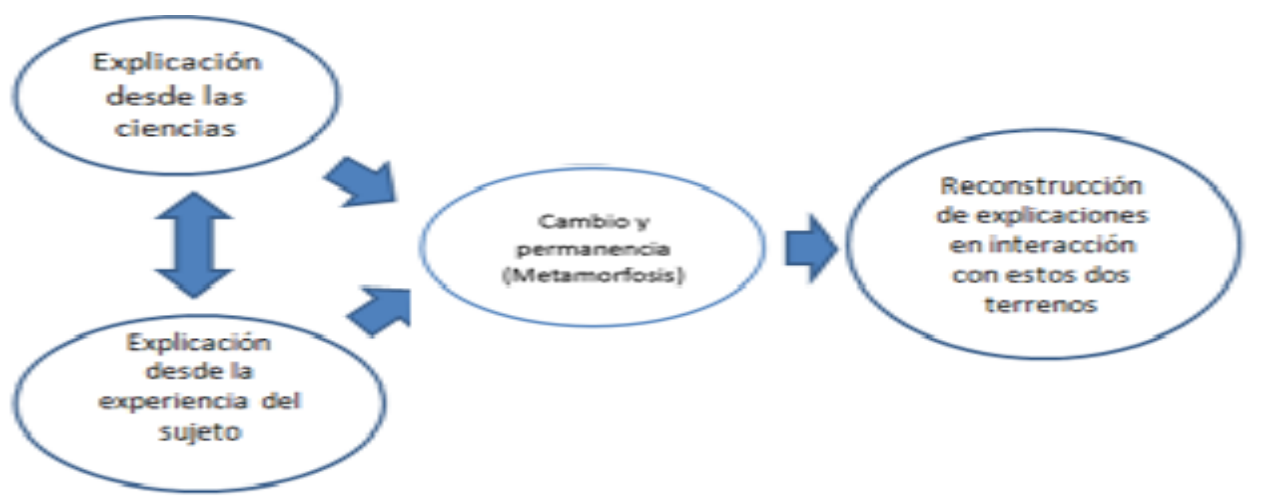

\section{Gráfico $\mathrm{N}^{\circ} 2$.}

Nótese la relación que tienen los dos terrenos que se mencionan a la hora de explicar el cambio y la permanencia de los organismos (explicaciones de la ciencia, explicaciones desde la experiencia del sujeto), estos dos terrenos son importantes para la reconstrucción y complejización de la explicación que se da respecto al fenómeno

Es importante para la enseñanza de las ciencias comprender el cambio y la permanencia de los organismos de una manera compleja y multicausal, propiciar espacios en donde el estudiante sea sujeto de conocimiento por medio del hacer y el comprender, estableciendo unas formas críticas y complejas de ver las condiciones en las que circulan sus propios saberes y los trabajos que se realizan desde las ciencias. 
Bio - grafia. Escritos sobre la Biología y su Enseñanza. ISSN 2027

Edición Extraordinaria. p.p. 1601-1613

Memorias del VIII Encuentro Nacional de Experiencias en Enseñanza de la Biología y la Educación Ambiental. III Congreso Nacional de Investigación en Enseñanza de la Biología.

Bibliografía.

Arca, M.; Guidoni, P.; Mazzolli, P. (1990). Enseñar ciencia. Cómo empezar: reflexiones para una educación científica de base. España: Paidós Educador.

Castro, A. (2005). La investigación del entorno natural: una estrategia didáctica para la enseñanza-aprendizaje de las ciencias naturales. Universidad Pedagógica Nacional.

Malagon, F. et al (2013) La actividad experimental: construcción de fenomenologías y procesos de formalización. Praxis Filosófica. No. 36, 119 - 138.

Mayr, E. (1995) Así es la biología. Argentina: Debate editorial.

Taylor y Bogdan (1996) Introducción a los métodos cualitativos. Paidos.

Jacob, F. (1984) La lógica de lo viviente. Barcelona: Salvat.

Jacob, F. (1998) El ratón, la mosca y el Hombre. Barcelona: Grigalbo.

Valencia, S., Méndez, O, Jiménez, G. Garzón, P. (2000). De la contemplación a la comprensión de los seres vivos. En: Revista Campo Abierto. Universidad de Extremadura España. N ${ }^{\circ} 20$.

Wilson, E. (2007) La creación: salvemos la vida en la Tierra. Buenos Aires: Katz Editores. 